\title{
Avaliação do ácido lático em indivíduos com hemiparesia pós-acidente vascular encefálico após estimulação elétrica para fortalecimento muscular Lactic acid assessment in post-stroke hemiparetic subjects following electrical stimulation for muscle strengthening
}

\author{
Fernanda Ishida Corrêa ${ }^{1}$, João Carlos Ferrari Corrêa ${ }^{2}$, André de Almeida Tessarolo ${ }^{3}$, André de Souza Melo ${ }^{3}$, \\ Luciana Maria Malosá Sampaioº, Maricilia Silva Costa ${ }^{4}$, Cláudia Santos Oliveira ${ }^{2}$
}

Estudo desenvolvido no Laboratório de Biodinâmica Humana da Uninove Universidade Nove de Julho, São Paulo, SP, Brasil

1 Fisioterapeuta; Profa. Ms. do Curso de Fisioterapia da Uninove

2 Profs. Drs. do Programa de Mestrado em Ciências da Reabilitação da Uninove

3 Graduandos em Fisioterapia na Uninove

4 Profa. Dra. do Curso de Engenharia Biomédica do Instituto de Pesquisa e Desenvolvimento da Universidade do Vale do Paraíba, São José dos Campos, SP

\section{ENDEREÇO PARA} CORRESPONDÊNCIA

Fernanda I. Corrêa Av. Francisco Matarazo, 612 Água Branca 05001-100 São Paulo SP e-mail: fecorrea@uninove.br

APRESENTAÇÃO abr. 2009

ACEITO PARA PUBLICAÇÃO jun. 2009
Resumo: O fortalecimento da musculatura parética promove melhora da capacidade funcional, o que pode ser obtido pela estimulação elétrica funcional (EEF), porém são necessários parâmetros específicos para evitar fadiga muscular. Este estudo visou verificar, em indivíduos com hemiparesia pós-AVE, o tempo de repouso (time off) necessário para reabsorção do ácido lático durante a EEF, de modo a evitar a fadiga - detectada por lactacidemia (concentração excessiva de ácido lático). Foram coletadas amostras de sangue de 18 indivíduos portadores de hemiparesia em sete momentos, antes, durante e após a EEF. A corrente quadrada bifásica, de $50 \mathrm{~Hz}$, foi usada com time on de 10 segundos (s) e o time off variou de $10 \mathrm{~s}$ (protocolo 1) a $30 \mathrm{~s}$ (protocolo 2), sobre o ponto motor do músculo tibial anterior. Na análise do sangue coletado, não foi encontrada diferença estatisticamente significante $(p>0,05)$ entre os dois protocolos; tampouco foi registrada diferença no período imediatamente pós-terapia. Pode-se pois afirmar que a ferramenta utilizada para mensurar lactacidemia não se mostrou eficaz na detecção da fadiga muscular, inviabilizando verificar o tempo ideal de repouso (time off), sendo necessários mais estudos em busca de protocolos seguros. Sugere-se a continuidade deste estudo, com alteração do músculo e atividade funcional eliciada pela estimulação elétrica, bem como uma ferramenta alternativa na mensuração e detecção da fadiga muscular.

DescritoRes: Acidose láctica; Fadiga muscular; Paresia; Terapia por estimulação elétrica

ABSTRACT: Strengthening paretic muscles improves functional capacity, which can be achieved by functional electric stimulation (FES), but specific parameters are needed in order to prevent muscle fatigue. The purpose of this study was to assess, in poststroke hemiparetic patients, the necessary time of rest (time off) for re-absorption of lactic acid during FES, so as to prevent fatigue - detected by lactic acid testing. Blood samples of 18 hemiparetic subjects were collected at seven moments: pretreatment rest, after each of three series of 15 repetitions stimuli, and at the third, sixth, and ninth minutes following therapy session. Electrical, two-phase pulses at $50 \mathrm{~Hz}$ were applied, 10 seconds on and 10 seconds off (protocol 1), and 10 seconds on and 30 seconds off (protocol 2), on the motor point of tibialis anterior muscle. The blood sample analysis showed no statistically significant differences $(p>0.05)$ between protocols 1 and 2; neither could differences be found at immediate postsession measures. It may thus be said that the measuring tool here employed to infer fatigue did not prove efficient to detect it, therefore preventing the assessment of the ideal time of rest (time off). Thus a continuity of this study is suggested, with changes in the muscle and the functional activity elicited by electric stimulation, and relying on an alternative tool for measuring and detecting muscle fatigue. Key words: Acidosis, lactic; Electrical stimulation therapy; Muscle fatigue; Paresis 


\section{INTRODUÇÃO}

O acidente vascular encefálico (AVE), responsabilizado como a terceira causa de morte nos Estados Unidos e por um terço dos óbitos no Brasil, é doença vascular caracterizada por um deficit neurológico focal, repentino e não-convulsivo, causado por lesão cerebral conseqüente a um mecanismo vascular não-traumático, por embolia arterial ou venosa, cursando com isquemia ou hemorragia cerebral ${ }^{1}$. A síndrome mais comumente vista após um AVE, síndrome do neurônio motor superior, é causada pelo infarto no território de irrigação da artéria cerebral média, levando ao acometimento do neurônio motor superior e de suas vias e conexões ${ }^{2,3}$. As seqüelas deixadas pelo AVE são variáveis e incluem alterações sensitivas, cognitivas e motoras, como fraqueza muscular ${ }^{4}$, espasticidade, padrões anormais de movimento ${ }^{5}$ e descondicionamento físico, incluindo fadiga muscular ${ }^{2,6}$.

Vários estudos ${ }^{7-9}$ têm utilizado a estimulação elétrica funcional (EEF) para diminuir essas alterações, mas os parâmetros para otimizar a força, enquanto simultaneamente minimizam a fadiga, não são tão bem conhecidos.

A EEF é uma técnica que produz contração muscular, despolarizando o nervo motor e produzindo uma resposta sincrônica em todas as unidades motoras do músculo. Esse sincronismo promove a contração eficiente, mas é necessário treinamento específico, a fim de se evitar a fadiga precoce, que impediria a utilização funcional do método com objetivos reabilitacionais ${ }^{10}$.

Não é possível obter um movimento funcional de um membro paralisado por um simples pulso elétrico; faz-se necessária uma série de estímulos, com certa duração, seguida por uma freqüência apropriada de repetição. Essa seqüência de estímulos recebe o nome de trem de $\operatorname{pulsos}^{2,7,11}$. Já foi relatado que freqüências variáveis no tempo, em torno de 20 a $40 \mathrm{~Hz}$, são mais efetivas para manter a força quando comparadas a freqüências contínuas, e, portanto, possibilitam o adiamento da fadiga precoce em pacientes pós-AVE².

Nos regimes de treinamento existe uma relação direta entre a intensidade da contração produzida eletricamente e o aumento da força muscular. Quanto maior a intensidade tolerada, maior será o número de unidades motoras recrutadas e maior a profundidade de ativação, a partir dos eletrodos de superfície ${ }^{12}$. Além disso, sobretudo quando se objetiva o fortalecimento muscular, a contração estimulada contínua do músculo esquelético leva à fadiga muscular muito rápida, o que implica queda da força gerada; é essencial então assegurar o estímulo de forma intermitente, mantendo uma taxa de repetição de trens de pulso, por meio dos controles time on (com estímulo) e time off (sem estímulo). O período time on de estimulação muscular para muitas aplicações é geralmente ajustado em até 10 ou 15 segundos; o time off é geralmente de 1 ou 2 minutos, sendo mais comumente praticado em 60 segundos ${ }^{2,8-10}$; tem sido sugerido pela literatura ${ }^{2,8-10}$ que esse intervalo, de aproximadamente 60 segundos, é necessário após contrações de 10 segundos de duração, para prevenir fadiga muscular durante o processo de fortalecimento com estimulação elétrica.

Como evidências ${ }^{10,11}$ sugerem que altas forças contráteis são necessárias para aumentar a força muscular (isto é, treiná-lo efetivamente), a geração desses níveis de contração apenas pode ser alcançada usando-se altas intensidades de estimulação, o que pode ser muito fatigante, levando a uma rápida queda de força se o time off for muito curto. $\mathrm{O}$ objetivo das aplicações da estimulação elétrica, quando se procura o fortalecimento muscular, é atingir o máximo de intensidade tolerável nas contrações. À medida que o tratamento é continuado, a amplitude de estimulação deve ser aumentada gradualmente até que o limiar motor seja alcançado e excedido ${ }^{10}$.

Por tudo isso, este estudo justifica-se com o objetivo de verificar se os tempos de repouso (time off) entre um estímulo elétrico e outro de 10 segundos (protocolo 1) e de 30 segundos (protocolo 2) são suficientes para evitar o aparecimento da fadiga muscular em indivíduos hemiparéticos por AVE; foi utilizada a dosagem de ácido lático como ferramenta de mensuração da fadiga muscular após a aplicação da terapia por estimulação elétrica funcional.
METODOLOGIA

Este foi um estudo transversal, cego e cruzado, no qual foram analisados pacientes portadores de hemiparesia em decorrência de AVE. A pesquisa foi aprovada pelo Comitê de Ética em Pesquisa da instituição. Todos os envolvidos foram informados e consentiram em participar do estudo por meio da assinatura de termo de consentimento livre e esclarecido.

Após cálculo estatístico da amostra $(n=18)$, foi realizada a triagem dos indivíduos portadores de hemiparesia em decorrência de AVE no Ambulatório de Neurologia da Universidade Nove de Julho, onde 29 pacientes com idades entre 35 e 50 anos foram avaliados. Não apresentar algum comprometimento associado que contra-indique o uso da estimulação elétrica, distúrbios de coagulação e distúrbio cognitivo que leve à dificuldade de compreensão foram outros critérios de inclusão. Após a triagem, foram selecionados 18 pacientes, que receberam explicações sobre a finalidade do estudo e orientações sobre os procedimentos a serem executados.

O programa de fortalecimento muscular por estimulação elétrica funcional, sobre o ponto motor do músculo tibial anterior, foi feito com um aparelho portátil (Quark VIF 475), de corrente quadrada e bifásica, freqüência de estimulação de $50 \mathrm{~Hz}$, período time on (t on) de 10 segundos, três séries de 15 contrações (repetições) com intervalo de 60 segundos entre cada série; a variável deste estudo foi o período time off ( $\mathrm{t}$ off) de 10 segundos (protocolo 1) e 30 segundos (protocolo 2). Entre os dois protocolos foi mantido um intervalo para repouso de uma semana, para evitar o efeito de somação da terapia anterior.

A fadiga muscular foi avaliada pela dosagem de ácido lático. Foram efetuadas coletas de sangue nos seguintes momentos: uma em repouso antes do tratamento; uma coleta após cada série de 15 repetições; e três coletas no 3으, 6음 e 9o minutos subseqüentes ao término da terapia. Para a coleta, foram utilizadas luvas cirúrgicas descartáveis e, após a assepsia com álcool da região do lóbulo da orelha, realizou-se a punção por meio de uma lanceta descartável. Desprezou-se a primeira gota de sangue para 
evitar sua contaminação com o lactato eliminado pelo suor. Em seguida, foram coletados 25 microlitros de sangue arterializado. Com o uso de capilares de vidro herarinizados e calibrados, o sangue coletado foi depositado em tubos (Ependorfs) contendo $50 \mu \mathrm{l}$ de fluoreto de sódio a $1 \%$ para evitar a continuação da glicólise.

Após coletadas, as amostras foram estocadas em freezer a $-100 \mathrm{C}$, posteriormente, analisadas por meio de um analisador de lactato pelo método eletroenzimático, com o aparelho YSI 1500 Sports (Yellow Springs Inc., Estados Unidos).

$\mathrm{Na}$ análise dos dados de concentração de lactato, foi aplicado o teste de Kolmogorov-Smirnov e observou-se distribuição normal para todos os dados. O nível de significância adotado foi de $5 \%$, sendo então estabelecido um a de 0,05 e um $ß$ de 0,20 . Como os dados foram paramétricos, o teste $t$ de Student foi utilizado para comparar a média dos dados entre os dois protocolos e o teste de comparação múltipla de médias DHS de Tukey, na presença de significância, para análise entre postos. O pacote estatístico utilizado foi o Instat.

\section{RESULTADOS}

Participaram deste estudo 18 indivíduos portadores de hemiparesia em decorrência de AVE. Na amostra inicial havia 29 pacientes, sendo que 11 foram excluídos, 4 por apresentarem doenças associadas que contra-indicavam o uso da estimulação elétrica, 3 com episódios de internação nas últimas quatro semanas que antecederam a avaliação, e 3 por não comparecerem na data marcada para avaliação. Os voluntários eram adultos, com idade entre 35 e 50 anos, sendo 10 homens e 8 mulheres, com média de idade semelhante entre os sexos, porém com massa e altura de $85,6 \mathrm{~kg}$ e 1,77 $\mathrm{cm}$ no sexo masculino e 65,16 kg e 1,59 $\mathrm{cm}$ no sexo feminino.

Conforme mostra a Tabela 1, os grupos mostraram-se semelhantes, sem diferença estatisticamente significante, em relação à coleta de repouso antes do tratamento e uma coleta após cada série de 15 repetições $(p<0,05)$.

Tabela 1 Concentração de lactato ( $\mathrm{mmmol} / \mathrm{L}$, média \pm desvio padrão), nas coletas em repouso (pré-tratamento) e nas coletas após cada série de 15 repetições, para os protocolos 1 e 2

\begin{tabular}{lcccc}
\hline Protocolo & $\begin{array}{c}\text { Repouso (pré- } \\
\text { tratamento) }\end{array}$ & Série 1 & Série 2 & Série 3 \\
\hline 1 ( off $10 \mathrm{~s})$ & $1,56 \pm 0,68$ & $1,18 \pm 0,53$ & $1,38 \pm 0,62)$ & $1,53 \pm 0,85$ \\
$2(\mathrm{t}$ off $30 \mathrm{~s})$ & $1,13 \pm 0,49$ & $1,25 \pm 0,48$ & $1,18 \pm 0,52$ & $1,23 \pm 0,51$ \\
\hline
\end{tabular}

Não foram encontradas diferenças estatisticamente significantes

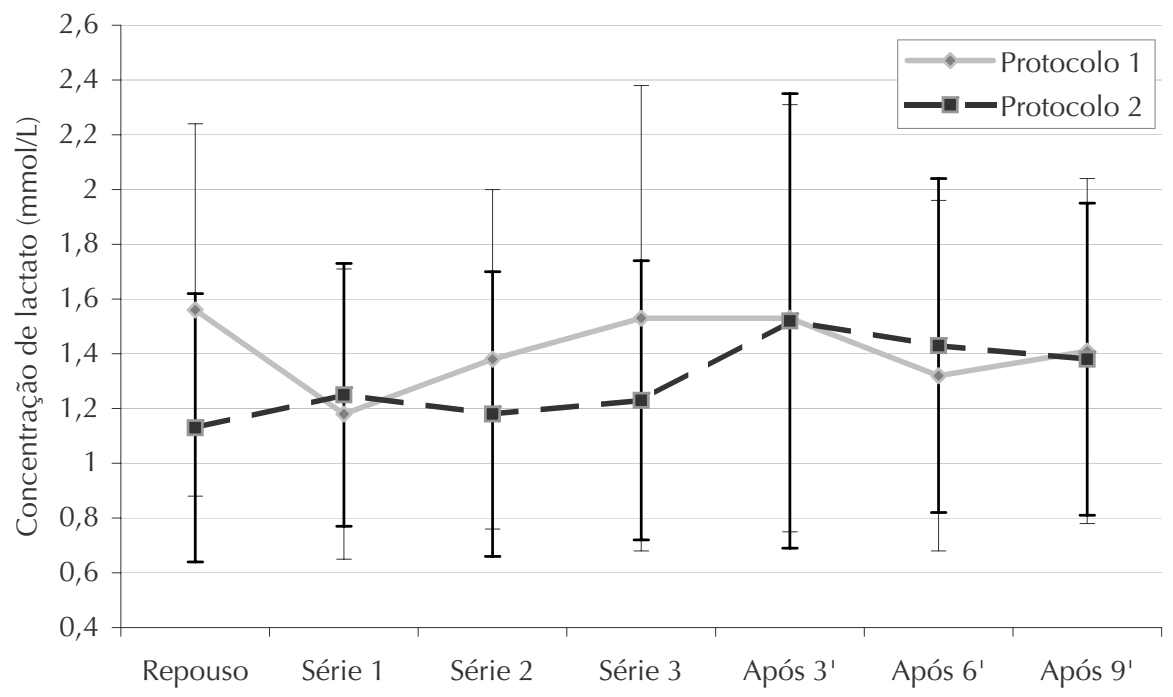

Gráfico 1 Concentração de lactato (média e desvio padrão) obtida em repouso (pré-tratamento), nas coletas após cada série de 15 repetições, e nas coletas no 3ำ 6o e 9o minutos subseqüentes ao término da terapia, nos protocolos 1 (t off de $10 \mathrm{seg}$ ) e 2 (t off de $30 \mathrm{seg}$ )

No Gráfico 1 pode-se observar que tanto no primeiro (t off de $10 \mathrm{seg}$ ) como no segundo protocolo (t off de $30 \mathrm{seg}$ ) não houve acúmulo de lactato que levasse ao limiar de anaerobiose, inferindo-se a possibilidade de o músculo não fadigar, nessas condições de aplicação da EEF.

\section{DISCUSSÃO}

Este estudo teve como principal objetivo verificar o tempo necessário de repouso, entre um estímulo elétrico e outro, para evitar o aparecimento da fadiga muscular em indivíduos hemiparéticos em decorrência de AVE; para isso, optamos pela utilização da dosagem de ácido lático (lactacidemia), como ferramenta de mensuração da fadiga muscular após utilização da terapia por estimulação elétrica funcional. De acordo com os resultados, pode-se afirmar que a ferramenta de mensuração utilizada, avaliação de lactacidemia, não se mostrou eficaz na detecção e mensuração da fadiga muscular do músculo tibial anterior, inviabilizando, conseqüentemente, verificar qual o tempo ideal de repouso (t off ), de 10 ou 30 segundos.

O músculo esquelético é composto por diferentes tipos de fibras. Sua estrutura histológica tem fibras musculares rápidas, lentas e, ainda, outras fibras com características intermediárias. Os músculos que reagem rapidamente são compostos, em sua grande maioria, por fibras rápidas (tipo II) com um número reduzido de fibras lentas (tipo I). Ao contrário, os que respondem de forma lenta com contrações longas, são compostos por uma maioria de fibras lentas. As fibras musculares rápidas apresentam grande quantidade de enzimas glicolíticas, menor número de mitocôndrias e suprimento sangüíneo menos extenso, visto que o metabolismo oxidativo tem importância secundária ${ }^{13,14}$.

As características metabólicas desses diferentes tipos de fibras exercem importante influência na fadigabilidade do músculo. A eletroestimulação crônica 
em músculos esqueléticos é capaz de provocar alterações importantes no perfil metabólico das fibras musculares, convertendo as fibras com características do tipo II em tipo I pelo aumento no volume mitocondrial, crescimento capilar, densidade capilar e suprimento de oxigênio. Dessa forma, esse recurso terapêutico aumenta a capacidade aeróbica oxidativa e a resistência à fadiga dos músculos isquêmicos ${ }^{12,14}$. Provoca, ainda, alterações na atividade das enzimas oxidativas, associada com a redução na atividade enzimática glicolítica ${ }^{13,14}$.

A fadiga surge após os exercícios nos quais se acumularam quantidades máximas de ácido lático; a recuperação plena implica a remoção desse ácido tanto do sangue quanto dos músculos esqueléticos que estiveram ativos durante o período precedente de exercícios ${ }^{15}$. Neste estudo, o ácido lático acumulado no sangue do sistema circulatório sistêmico, pela análise do lactato, não evidenciou alteração alguma que denotasse o aparecimento da fadiga muscular causada pela estimulação elétrica funcional localizada em um músculo do membro inferior. Pode-se sugerir que tal fadiga, se gerada pela estimulação elétrica no músculo especificamente, não é suficiente para registrar qualquer diferença na dosagem de lactato sistemicamente.

O ácido lático acumulado no sangue e nos músculos durante o exercício aeróbio e anaeróbio é removido durante o período de recuperação. A velocidade dessa remoção depende do fato de se ficar em repouso durante a recuperação (repouso-recuperação) ou de realizar um exercício leve (30 a $65 \%$ do VO máximo) durante a recuperação (exercício-recuperação). O ácido lático é removido mais rapidamente durante o exercício-recuperação, e seu destino principal é a oxidação, processada principalmente no músculo esquelético, mas que também ocorre nos tecidos cardíaco, renal, hepático e cerebral e pelo sistema tampão ${ }^{15}$. Os resultados deste estudo não permitem avaliar se esse pro- cesso de remoção se deu no protocolo de estimulação elétrica realizado, conforme descrito na literatura, devido à não-detecção e evidenciação do aumento de ácido lático acumulado no sangue sistêmico.

A literatura demonstra que a ativação muscular por eletroestimulação neuromuscular ativa primeiramente as fibras musculares do tipo Ilb, que são fibras "brancas" de contração rápida, glicolíticas, que possuem poucas redes de capilares e poucas mitocôndrias, utilizando principalmente o metabolismo anaeróbio para a produção de energia e assim realizar a contração muscular. A utilização do metabolismo anaeróbio para a síntese de ATP (trifosfato de adenosina) faz com que ocorra um aumento da produção de ácido lático; quando essa produção é maior que sua reabsorção, ocorre o fenômeno chamado acidose metabólica; com isso, ocorre uma diminuição das reações enzimáticas, o que gera a diminuição da liberação de cálcio, que estimula a troponina e tropomiosina, não ocorrendo a formação da ponte cruzada; assim, os filamentos de miosina não se interligam com os filamentos de actina para realizar a contração muscular, ou seja, geram fadiga muscular ${ }^{16,17}$.

Considerando pesquisas anteriores, que citam o intervalo de 60 segundos como o tempo necessário para prevenir a fadiga muscular durante o processo de eletroestimulação $\mathrm{O}^{2,8-10,18}$, buscou-se com este trabalho investigar dois diferentes protocolos de eletroestimulação, com dois diferentes parâmetros do t off (10 e 30 segundos). Ambos podem ter se mostrado eficazes, pois todos os pacientes que participaram da pesquisa não apresentaram aumento do ácido lático ou manifestações de fadiga muscular; mas também se pode inferir o não-aumento do ácido lático na ferramenta de mensuração utilizada neste estudo, como já discutido. Não foi investigado o tempo de 60 segundos de t off porque o apareIho de estimulação elétrica utilizado não permitia esse intervalo de descanso para um t on fixado previamente em 10 seg.
A escolha dos outros parâmetros, como a freqüência de $50 \mathrm{~Hz}$, aconteceu por ser a freqüência mais utilizada pelos estudos ${ }^{19-22}$, que utilizaram a EENM para fortalecimento muscular, inclusive em portadores de hemiparesia, com resultados positivos para fortalecimento. Contudo, em outro estudo ${ }^{18}$ que analisou o efeito da eletro estimulação com duas freqüências diferentes, baixa (50 $\mathrm{Hz}$ ) e média freqüência $(2000 \mathrm{~Hz})$, avaliando a fadiga pela eletromiografia, mostrou que o grupo estimulado com baixa freqüência gerou maior fadiga muscular que o grupo estimulado com média freqüência, portanto, a EENM com corrente de média freqüência mostrou-se mais vantajosa, neste estudo.

Em virtude de nossos limitados resultados, além de outras divergências acima relatadas, e sabendo da importância da eletroestimulação como coadjuvante na melhora funcional dos pacientes hemiparéticos - com aumento da amplitude de movimento, diminuição da espasticidade e aumento de força muscular -, tornam-se necessários mais estudos em busca de protocolos seguros. Assim sugere-se uma continuidade deste estudo, com alteração do músculo e atividade funcional eliciada pela estimulação elétrica, bem como uma ferramenta alternativa para mensuração e detecção da fadiga muscular.

\section{CONCLUSÃO}

Os resultados permitem afirmar que a ferramenta de mensuração utilizada neste estudo, lactacidemia, não se mostrou eficaz na detecção e mensuração da fadiga muscular do músculo tibial anterior, uma vez que o ácido lático acumulado no sangue do sistema circulatório sistêmico, pela análise do lactato, não evidenciou nenhuma alteração que denotasse o aparecimento da fadiga muscular. Pode-se sugerir que tal fadiga, se gerada pela estimulação elétrica no músculo especificamente, não é suficiente para registrar qualquer diferença na dosagem de lactato sistemicamente. 


\section{REFERENCIAS}

1 Ladeia ML, Guimarães AC. Doença cerebrovascular. Rev Neuropsiquiatria. 2003;6(1):54-61.

2 Doucet BM, Griffin L. Variable stimulation patterns for poststroke hemiplegia. Muscle Nerve. 2009;39(1):54-62.

3 Carr JH, Shepherd RB, Ada L. Spasticity: research findings and implications for intervention. Phisiotherapy. 1995:81(8):421-9.

4 Teixeira-Salmela LF, Olney SJ, Nadeau S, Brouwer B. Muscle strengthening and physical conditioning to reduce impairment and disability un chronic stroke survivors. Arch Phys Med Rehabil. 1999;80:1211-8.

5 Canning CG, Ada L, O'Dwyer NJ. Abnormal muscle activation characteristics associated with loss of dexterity after stroke. J Neurological Sci. 2000;176:45-56.

6 Allen DG. Fatigue in working muscles. J Appl Physiol. 2009;106(2):378-84.

7 Gauche E, Couturier A, Lepers R, Michaut A, Rabita G, Hausswirth C. Neuromuscular fatigue following high versus low-intensity eccentric exercise of biceps brachii muscle. J Electromyogr Kinesiol. 2009; [epub ahead of print].

8 Vassilakos G, James RS, Cox VM. Effect of stimulation frequency on force, net power output, and fatigue in mouse soleus muscle in vitro. Can J Physiol Pharmacol. 2009;87(3):203-10.

9 Braz GP, Russold M, Smith RM, Davis GM. Efficacy and stability performance of traditional versus motion sensorassisted strategies for FES standing. J Biomech. 2009; [epub ahead of print].

10 Durfee WK. Electrical stimulation for restoration of function. NeuroRehabilitation. 1999;12:53-62.

11 Mount J, Dacko S. Effects of dorsiflexor endurance exercises on foot drop secondary to multiple sclerosis: a pilot study. NeuroRehabilitation. 2006;21:43-50.

12 Lianza, S. Estimulação elétrica funcional [tese livre docência]. Rio de Janeiro: Universidade Federal do Rio de Janeiro; 1990.
13 Guyton AC, Hall JE. Tratado de fisiologia médica. Rio de Janeiro: Guanabara Koogan; 2002. Cap. 6 Contração do músculo esquelético. p.63-74.

14 Hamilton MT, Booth FW. Skeletal muscle adaptation to exercise: a century of progress. J Appl Physiol. 2000;88:327-31.

15 Filus R. O efeito do tempo de rodízios entre postos de trabalho nos indicadores de fadiga muscular: o ácido lático [dissertação]. Curitiba: Faculdade de Engenharia Mecânica, Universidade Federal do Paraná; 2006.

16 Sampaio LMM. Adaptações fisiológicas do paciente asmático ao exercício físico [tese]. São Carlos: Faculdade de Ciências Biológicas e da Saúde, Universidade Federal de São Carlos; 2002.

17 Gonçalves M. Eletromiografia e a identificação da fadiga muscular. Rev Bras Educ Fisica. 2006;20(5):91-3

18 Pires KF. Análise dos efeitos de diferentes protocolos de eletroestimulação neuromuscular através da freqüência mediana. Rev Bras Cienc Mov. 2004;12(2):25-8.

19 Costa JRC, Volpe MAS, Jorge S. Efeito da estimulação elétrica neuromuscular sobre grupo extensor de punho em paciente com seqüela de AVE: relato de caso. Arq Cienc Saude Unipar. 2004;8(2):129-34.

20 Schuster RC, Sant CR, Dalbosco V. Efeitos da estimulação elétrica funcional (FES) sobre o padrão de marcha de um paciente hemiparético. Acta Fisiatr. 2007;14(2):82-6.

21 Nunes LCBG, Quevedo AAF. Efeitos da eletroestimulação neuromuscular no músculo tibial anterior de pacientes hemiparéticos espásticos [dissertação]. Campinas: Faculdade de Engenharia Elétrica e Computação, Universidade Estadual de Campinas; 2004.

22 Borrani F, Malatesta D, Candau R. Is a progressive recruitment of muscle fibers required for the development of the slow component of VO2 kinetics? J Appl Physiol. 2008;105(2):575-80. 